\title{
Dynamic changes of the fecal bacterial community in dairy cows during early lactation
}

\author{
Shuai Huang ${ }^{1} \mathbb{D}$, Shoukun $\mathrm{Ji}^{2}$, Feiran Wang ${ }^{1}$, Jie Huang ${ }^{3}$, Gibson Maswayi Alugongo ${ }^{1}$ and Shengli $\mathrm{Li}^{1 *}$ (1)
}

\begin{abstract}
The dynamics of the community structure and composition of the dairy cow fecal bacterial communities during early lactation is unclear, therefore this study was conducted to characterize the fecal bacterial communities in dairy cows during early lactation using $16 \mathrm{~S}$ rRNA gene sequencing. Feces were sampled from 20 healthy fresh Holstein dairy cows on day 1 (Fresh1d group) and day 14 (Fresh14d group) after calving. After calving, cows were fed the same fresh diet. The dominant phyla Firmicutes and Proteobacteria were decreased $(P \leq 0.01)$ with lactating progress and phyla Bacteroidetes were increased $(P=0.008)$ with lactating progress and dietary transition. At family level, the predominant families were Ruminococcaceae (35.23\%), Lachnospiraceae (11.46\%), Rikenellaceae (10.44\%) and Prevotellaceae (6.89\%). A total of 14 genera were different between fecal samples from Fresh1d and Fresh14d, included the predominant genera, such as Ruminococcaceae_UCG-005 $(P=0.008)$, Rikenellaceae_RC9_gut_group $(P=0.043)$ and Christensenellaceae_R-7_group $(P=0.008)$. All fecal bacterial communities shared members of the genera Ruminococcaceae_UCG-005, Bacteroides and Rikenellaceae_RC9_gut_group. These findings help to improve our understanding of the composition and structure of the fecal microbial community in fresh cows and may provide insight into bacterial adaptation time and dietary in lactating cows.
\end{abstract}

Keywords: Fecal microbiota, Fresh dairy cows, Dynamic, 165 rRNA sequencing

\section{Introduction}

The bovine gastrointestinal tract microbiota harbors lots of microbial species that play important roles in the health and productivity of ruminant (Bergmann 2017; Clemmons et al. 2019; Myer et al. 2015). These microbes are necessary for fermentation of ingested plant matter into compounds such as volatile fatty acids that act as energy sources for the host (Flint et al. 2008). The development of high-throughput sequencing technology has enabled advancements in the understanding of the gastrointestinal microbiota of ruminant animals in recent years. Studies on the microbiota present in rumen during

\footnotetext{
*Correspondence: lisheng0677@163.com

${ }^{1}$ The State Key Laboratory of Animal Nutrition, Beijing Engineering Technology Research Center of Raw Milk Quality and Safety Control, College of Animal Science and Technology, China Agricultural University, Beijing 100193, China

Full list of author information is available at the end of the article.
}

lactation have found that the ruminal microbial structure and composition varied with lactation period (Bainbridge et al. 2016; Lima et al. 2015; Xue et al. 2018), and revealed an association between the rumen microbiota and milk efficiency (Weimer et al. 2017), feed efficiency (Shabat et al. 2016) and milk production (Indugu et al. 2017; Tong et al. 2018) in dairy cows.

In addition to studies on the rumen, reports on the microbial composition of the feces in dairy calves are abundant. Previous studies have found an association between fecal microbiota and age (Song et al. 2018), diet (Dill-McFarland et al. 2019; Wang et al. 2019), antibiotic therapy (Behr et al. 2018; Oultram et al. 2015; Yousif et al. 2018), and health (Gomez et al. 2017) in dairy calves. For lactating cows, the core fecal microbiota was identified from ten farms across Northern and Central California, USA (Hagey et al. 2019). Another study compared the fecal microbiota of health and left-sided displacement of the abomasum and found a shift in fecal microbiota 
composition with left-sided displacement in early lactating dairy cows (Song et al. 2016). Therefore, to date, the knowledge on the fecal microbiota structure and composition during early lactation stage in dairy cows, remain sparse.

It is well known that diet can make a significantly influence in the structure of the fecal microbiome (Kim et al. 2014; Zhang et al. 2018). However, lactation was found to be another important factor to shape rumen microbiome in dairy cows (Bainbridge et al. 2016). For fecal microbiota, limited knowledge about the diet and lactating effect on fecal microbiota. Therefore, elucidating the dynamic changes of fecal microbiota in dairy cows is expected to enable improvements to feed and management strategies for dairy cows, especially in the fresh period, which is the most sensitive window for dairy cows. Here, we aimed to characterize the community structure and composition of the fecal microbiota in dairy cows during early lactation stage. Our findings may contribute to the state of knowledge on the hindgut bacterial community structure in lactating dairy cows and identify genera of interest for further studies into the functional roles of the fecal microbiota in the health of the host.

\section{Materials and methods}

\section{Cows and management}

Twenty fresh (2.48 \pm 0.59 parity) Holstein dairy cows were housed in a free-stall barn at a commercial dairy farm (Beijing, China). The dietary and nutritional composition of the feed given to fresh cows is presented in Additional file 1: Table S1. Cows were allowed ad libitum access to feed and fresh water.

\section{Collection of fecal samples}

Forty fecal samples were collected from 20 healthy fresh cows on $\mathrm{d} 1$ and $\mathrm{d} 14$ after calving, without a history of antibiotic or drug treatment for 3 months prior to collecting samples. Feces were collected by hand from the rectum of cows using sterilized gloves before morning feeding. All samples were immediately transported on liquid nitrogen and later stored at $-80{ }^{\circ} \mathrm{C}$ before DNA extraction.

\section{Fecal bacteria DNA extraction, amplification and sequencing}

Total DNA was extracted from all fecal samples using an Omega Stool DNA kit (Omega Bio-Tek, Norcross, GA, USA) according to the manufacturer's instructions. Amplicon library preparation was performed by PCR amplification of the V3 to V4 region of the 16S rRNA gene, using forward (338F, 5'-ACTCCTACGGGAGGC AGCAG-3') and reverse primers (306R, 5'-GGACTA CHVGGGTWTCTAAT-3'). Then, the amplicon library was sequenced using the Illumina Miseq platform (Illumina, San Diego, CA, USA) by Beijing Allwegene Tech. Ltd (Beijing, China). A $25 \mu \mathrm{L}$ reaction mixture containing $12.5 \mu \mathrm{L}$ of KAPA $2 \mathrm{G}$ Robust Hot Start Ready Mix (Kapa Biosystems, Wilmington, MA, USA), $1 \mu \mathrm{L}$ of each

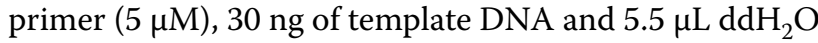
was used for PCR in triplicate, with the following cycling conditions: $95{ }^{\circ} \mathrm{C}$ for $5 \mathrm{~min}$ in denaturation, followed by 28 cycles of $95{ }^{\circ} \mathrm{C}$ for $45 \mathrm{~s}$ in denaturation, $55^{\circ} \mathrm{C}$ for $50 \mathrm{~s}$ in annealing and $72{ }^{\circ} \mathrm{C}$ for $45 \mathrm{~s}$ in elongation at with a final extension at $72{ }^{\circ} \mathrm{C}$ for $10 \mathrm{~min}$.

Amplicons were detected by $1 \%$ agarose gel electrophoresis and purified using the Agencourt AM Pure XP Kit (Beckman Coulter Genomics, Indianapolis, IN, USA). Purified amplicons from samples were quantified using Caliper LabChip GX Touch HT (PerkinElmer, Downers Grove, IL, USA), pooled in equimolar concentrations into the final library, and then $2 \times 250$ paired-end sequenced on an Illumina MiSeq platform (Caporaso et al. 2012).

\section{Cleanup of sequencing data}

High-quality sequence extraction was first conducted with Quantitative Insights into Microbial Ecology (QIIME) version 1.8.0 (Caporaso et al. 2010). Raw FASTQ files were de-multiplexed and quality-filtered with the following criteria: (I) reads with an average quality score of less than 20 were removed; (II) reads that did not exactly match to primer sequences and barcode tags and reads containing ambiguous characters were removed; (III) only overlapping sequences longer than $10 \mathrm{bp}$, and reads less than $230 \mathrm{bp}$ after overlapping were assembled; (IV) reads that could not be assembled were removed. The sequences were classified into operational taxonomic units (OTUs) under the threshold of 97\% identity using USEARCH (version 10.0.240) after removing singletons, and chimers were identified and removed using UCHIME (Edgar 2013). The most abundant sequence within each OTU from specific libraries (libraries constructed for bacteria) was designated as the "representative sequence"; this sequence was then aligned against the SILVA 128 16S rRNA gene database (Pruesse et al. 2007), with a confidence threshold of $70 \%$, using the Ribosomal Database Project Classifier (Wang et al. 2007).

\section{Statistical analysis of microbiota}

Alpha diversity indices (Chao1 value, number of OTUs, Shannon and Simpson indices) were assessed with QIIME 1.8. Differences in community richness and diversity were analyzed with the Kruskal-Wallis test, with $P$ values corrected by false discovery rate (FDR) from multiple comparisons in package "ggpurb" of $\mathrm{R}$ 3.6.2 (R, Armonk, NY, USA). The none-metric multidimensional scaling (NMDS) plot of the microbial profiles was 
performed based on Bray-Curtis distance (calculated by QIIME) using the "vegan" package in R (Oksanen et al. 2015). Analysis of similarity (ANOSIM) for multivariate data was calculated using the Bray-Curtis distance metric within the function of "vegdist" and "anosim" in the R package "vegan".

Comparison of the microbial structure and composition at phylum and genus levels were performed using the Wilcoxon tests with the FDR correlation, as described by Benjamini and Hochberg (1995) for pairwise comparisons. The false discovery rate corrected $P$ values $\leq 0.05$ were considered to indicate significant differences.

\section{Nucleotide sequence accession numbers}

All the raw DNA sequences were deposited in the National Center for Biotechnology Information Sequence Read Archive database and are publicly accessible under the accession number PRJNA628713.

\section{Results}

Sequencing, evenness and richness of the fecal microbiota Bacterial amplicons for all fecal samples were sequenced, and a total of 1,100,195 raw reads were generated; of these, 1,076,491 high-quality reads were obtained from all the 40 samples. After sub-sampling and clustering, $1000 \pm 144$ OTUs were identified. The Good's coverage for each sample was deemed sufficient, with values $>99.00 \%$ for all bacterial communities, implying that the current sequencing depth was sufficient to be representative of the microbiota studied (Table 1). During early lactation stage, a significant decrease $(P<0.05)$ in OTU number and Chao 1 was observed from $\mathrm{d} 1$ to $\mathrm{d} 14$, indicating significant changes in the diversity and richness of fecal microbiota as lactation progressed (Table 1).

NMDS based on Bray-Curtis distance showed a separation between Fresh1d and Fresh14d groups (Fig. 1). We further performed ANOSIM to demonstrate the effect of the lactation stage. The ANOSIM results revealed a

Table 1 Number of operational taxonomic units (OTU), Good's coverage, Chao1 and Shannon indices for fecal samples obtained at $d 1$ and d14 in fresh cows after calving

\begin{tabular}{lllll}
\hline Indices & \multicolumn{2}{l}{ Stage } & SEM & P value \\
\cline { 2 - 3 } & Fresh1d & Fresh14d & & \\
\hline OTU & 1060 & 940 & 22.38 & 0.026 \\
Good's coverage & 0.991 & 0.992 & 0.0002 & 0.026 \\
Chao1 & 1254.05 & 1125.12 & 25.30 & 0.026 \\
Shannon index & 7.76 & 7.61 & 0.07 & 0.440 \\
\hline
\end{tabular}

Fresh1d indicates fecal microbiota samples from cows on d1, Fresh14d indicates fecal microbiota samples from cows on d14

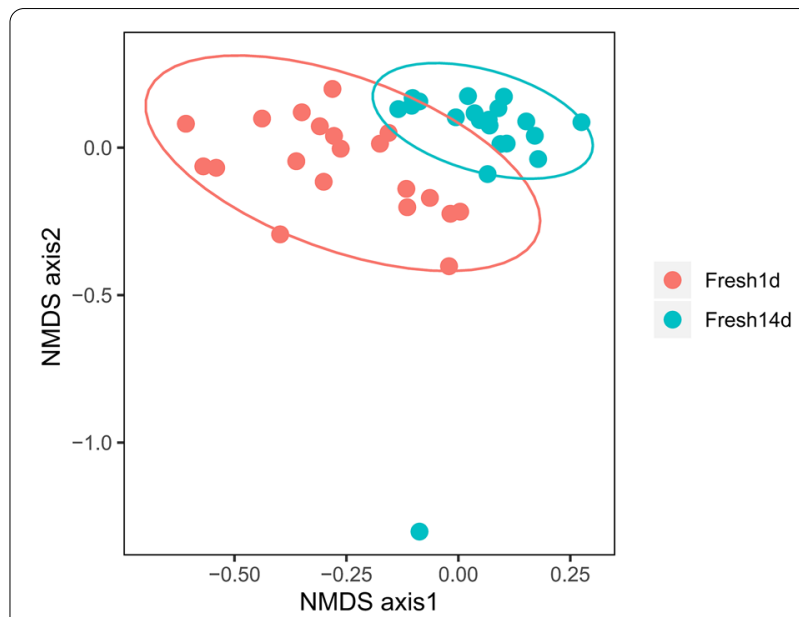

Fig. 1 None-metric multidimensional scale analysis based on Bray-Curtis distance. ANOSIM analysis of the samples on $\mathrm{d} 1$ and d14, where bacterial communities in dairy cow feces are grouped by lactation period in feces. Analysis was conducted using a Bray-Curtis metric based on operational taxonomic units $\left(R^{2}=0.447, P=0.001\right)$. Fresh1d indicates fecal microbiota samples from cows on $\mathrm{d} 1$, Fresh14d indicates fecal microbiota samples from cows on d14

significant difference in fecal bacterial community composition between Fresh1d and Fresh14d $\left(R^{2}=0.447\right.$, $P=0.001)$.

\section{Taxonomic composition of the fecal microbiota}

In total, 17 phyla were identified; among these, Firmicutes, Bacteroidetes, Proteobacteria and Spirochaetae were the predominant phyla across all samples, representing $63.69,29.65,1.60$ and $1.26 \%$ of total sequences, respectively (Fig. 2a). Two other phyla, namely Actinobacteria and Saccharibacteria, accounted for $2.34 \%$ of the community and were considered minor contributing phyla (Fig. 2a, b, Table 2).

Among all identified families, the predominant family included Ruminococcaceae (35.23\%), Lachnospiraceae (11.46\%), Rikenellaceae (10.44\%) and Prevotellaceae (6.89\%, Fig. 2a, b). The relative abundance $>2 \%$ across samples were Christensenellaceae (4.76\%), Bacteroidaceae (3.85\%), Peptostreptococcaceae (3.36\%), Family_XIII (2.32\%), Bacteroidales_S24-7_group (2.24\%) and Bacteroidales_RF16_group (2.21\%), and altogether comprised $18.74 \%$ of total samples.

Of the 217 genera identified, Ruminococcaceae UCG-005 (14.90\%), Rikenellaceae_RC9_gut_group (7.52\%), Ruminococcaceae_UCG-010 (4.94\%), Christensenellaceae_R-7_group (4.65\%), Eubacterium_ coprostanoligenes_group (4.02\%), Bacteroides (3.85\%), Prevotellaceae_UCG-003 (3.55\%), Alistipes (2.50\%), Ruminococcaceae_UCG-013 (2.42\%), Romboutsia 
a
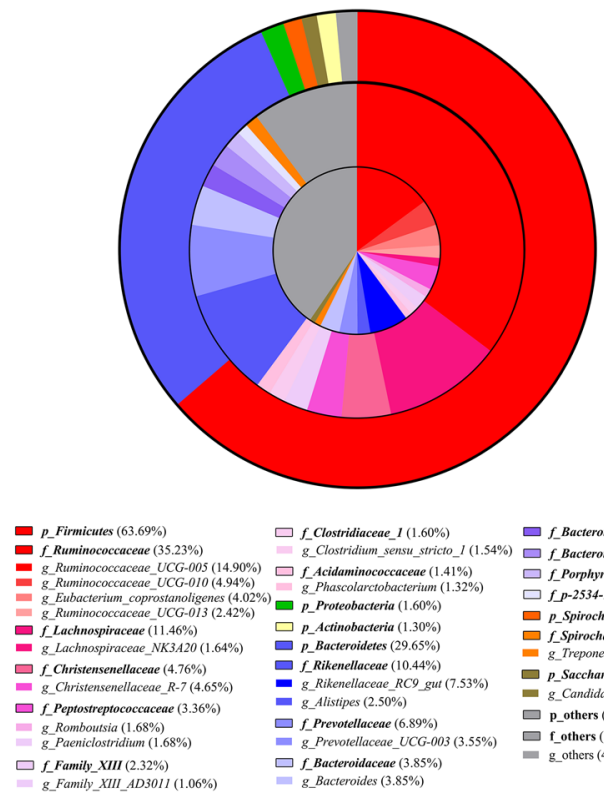

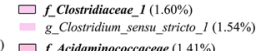

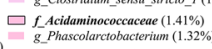

D-Proteobacteria $(1.60 \%)$

D_Actinobacteria $(1.30 \%)$

f_Rikenellaceae $(10.44 \%)$

=.

$\square$ f Prevotellaceae $(6.89 \%)$

g_Prevotellaceae_UCG-003

$\square \underset{g_{-} \text {Bacterorides }(3.85 \%)}{ }$
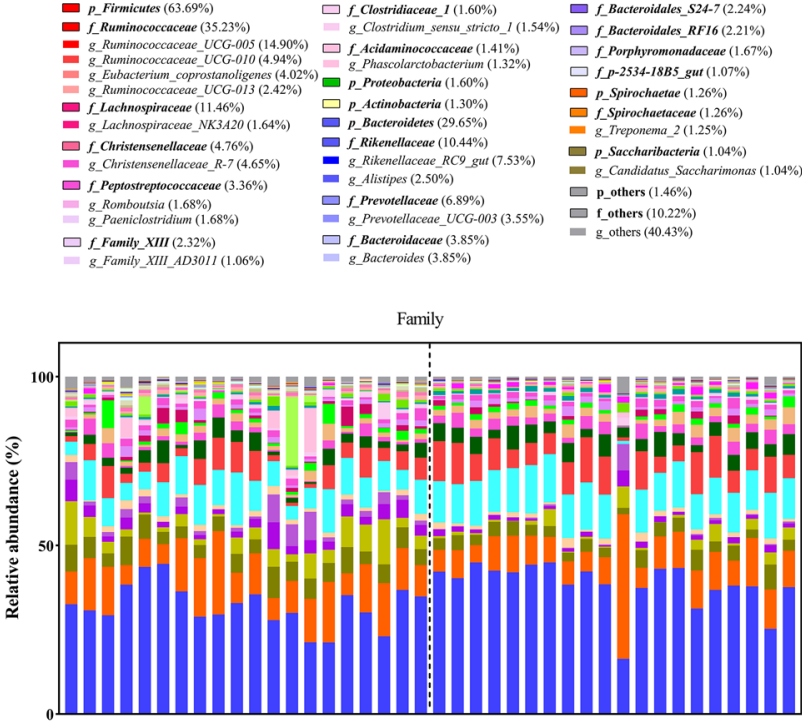

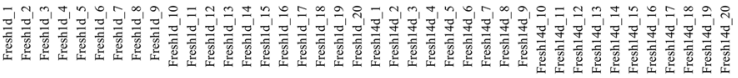
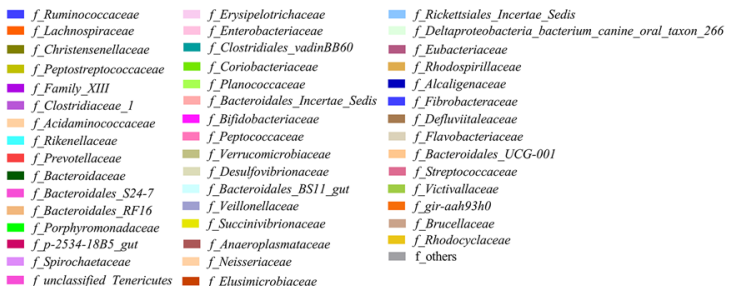

b
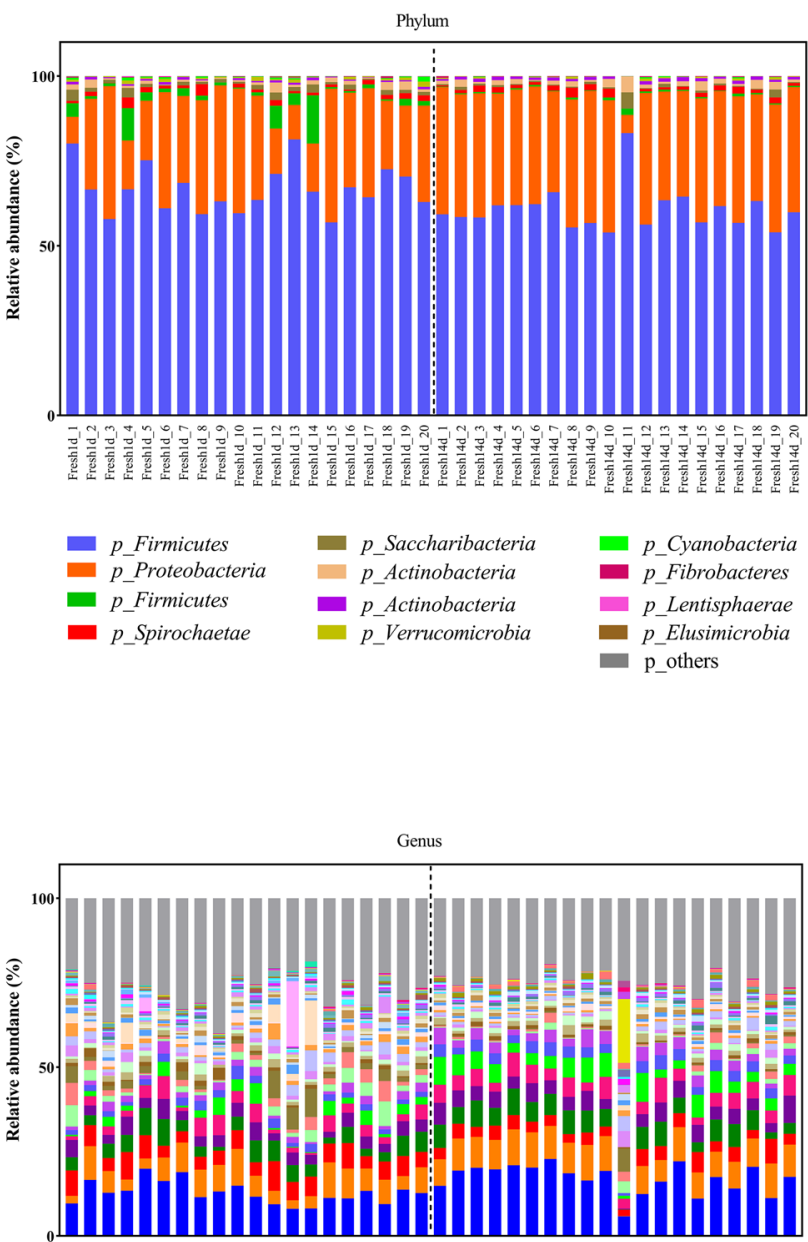

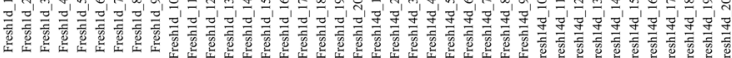
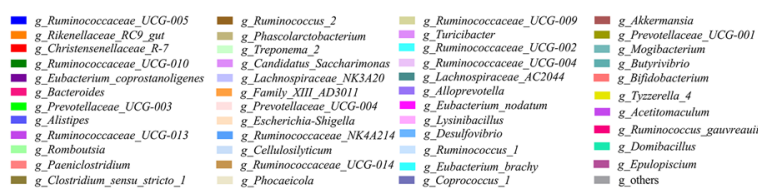

Fig. 2 The relative abundance of fecal samples at the phylum, family and genus level. a Distribution of predominant phyla, family and genera of all fecal samples (relative abundance $>1 \%$ at all samples). b The fecal bacterial community composition of all animals at the phyla, family and genus level (relative abundance $>1 \%$ at least one sample). Fresh1d indicates fecal microbiota samples from cows on d1, Fresh14d indicates fecal microbiota samples from cows on d14

(1.68\%), Paeniclostridium (1.68\%), Lachnospiraceae NK3A20_group (1.64\%), Clostridium_sensu_stricto_1 (1.54\%), Phascolarctobacterium (1.32\%), Treponema_2 (1.25\%), Family_XIII_AD3011_group (1.06\%), and Candidatus_Saccharimonas (1.04\%) were the predominant genera (17 in total) and altogether comprised $59.57 \%$ of the fecal community (Fig. 2a, b, Table 3).

\section{Shared OTUs and core fecal bacteria in fresh cows}

Among the total number of OTUs, 116 OTUs were shared by all samples, representing a total relative 
Table 2 Effect of lactation period on diversity at the phylum level in the fecal bacterial community

\begin{tabular}{lllrl}
\hline Phyla & \multicolumn{2}{l}{ Stage } & SEM & P value \\
\cline { 2 - 3 } & Fresh1d & Fresh14d & & \\
\hline Firmicutes & 66.70 & 60.68 & 0.06 & 0.010 \\
Bacteroidetes & 25.38 & 33.93 & 1.52 & 0.008 \\
Proteobacteria & 2.73 & 0.46 & 0.01 & 0.003 \\
Saccharibacteria & 1.30 & 0.78 & $<0.01$ & 0.025 \\
Spirochaetae & 1.21 & 1.32 & $<0.01$ & 0.780 \\
Actinobacteria & 1.08 & 1.51 & 0.91 & 0.067 \\
Verrucomicrobia & 0.54 & 0.26 & $<0.01$ & 0.067 \\
Tenericutes & 0.52 & 0.84 & $<0.01$ & 0.003 \\
Cyanobacteria & 0.32 & 0.09 & 0.09 & 0.013 \\
Fibrobacteres & 0.09 & 0.10 & 0.05 & 0.380 \\
\hline
\end{tabular}

Fresh1d indicates fecal microbiota samples from cows on d1, Fresh14d indicates fecal microbiota samples from cows on $\mathrm{d} 14$

Table 3 Effect of lactation period on diversity at the genus level in the fecal bacterial community

\begin{tabular}{|c|c|c|c|c|}
\hline \multirow[t]{2}{*}{ Genera } & \multicolumn{2}{|l|}{ Stage } & \multirow[t]{2}{*}{ SEM } & \multirow[t]{2}{*}{$P$ value } \\
\hline & Fresh1d & Fresh14d & & \\
\hline Ruminococcaceae_UCG-005 & 12.78 & 17.02 & 0.69 & 0.008 \\
\hline Rikenellaceae_RC9_gut_group & 6.44 & 8.61 & 0.43 & 0.043 \\
\hline Christensenellaceae_R-7_group & 5.49 & 3.81 & 0.27 & 0.008 \\
\hline Ruminococcaceae_UCG-010 & 4.41 & 5.46 & 0.32 & 0.17 \\
\hline $\begin{array}{l}\text { Eubacterium_coprostanoligenes_ } \\
\text { group }\end{array}$ & 3.60 & 4.44 & 0.23 & 0.074 \\
\hline Bacteroides & 3.13 & 4.58 & 0.29 & 0.064 \\
\hline Romboutsia & 2.51 & 0.86 & 0.26 & 0.008 \\
\hline Paeniclostridium & 2.33 & 1.02 & 0.26 & 0.043 \\
\hline Prevotellaceae_UCG-003 & 2.29 & 4.81 & 0.37 & 0.013 \\
\hline Alistipes & 1.98 & 3.03 & 0.14 & 0.012 \\
\hline Lachnospiraceae_NK3A20_group & 1.95 & 1.34 & 0.20 & 0.033 \\
\hline Ruminococcaceae_UCG-013 & 1.86 & 2.98 & 0.19 & 0.048 \\
\hline Family_XIII_AD3011_group & 1.53 & 0.58 & 0.14 & 0.004 \\
\hline Phascolarctobacterium & 1.35 & 1.30 & 0.10 & 1.000 \\
\hline Candidatus_Saccharimonas & 1.30 & 0.78 & 0.15 & 0.048 \\
\hline Treponema_2 & 1.19 & 1.31 & 0.49 & 0.008 \\
\hline Ruminococcus_2 & 1.14 & 0.77 & 0.12 & 0.860 \\
\hline $\begin{array}{l}\text { Ruminococcaceae_NK4A214_ } \\
\text { group }\end{array}$ & 1.10 & 0.67 & 0.10 & 0.190 \\
\hline Turicibacter & 1.01 & 0.33 & 0.08 & 0.019 \\
\hline Ruminococcaceae_UCG-014 & 0.87 & 0.95 & 0.14 & 0.009 \\
\hline Lachnospiraceae_AC2044_group & 0.51 & 0.74 & 0.08 & 0.550 \\
\hline Tyzzerella_4 & 0.16 & 1.08 & 0.07 & 0.200 \\
\hline
\end{tabular}

Fresh1d indicates fecal microbiota samples from cows on d1, Fresh14d indicates fecal microbiota samples from cows on d14 abundance of $49.82 \%$ across samples (Additional file 1: Table S2). At the family level, 18 classified families shared across all samples (Additional file 1: Table S2); of them, the relative abundance $>3 \%$ across samples were Ruminococcaceae (18.00\%), Lachnospiraceae (6.52\%), Rikenellaceae (4.03\%), Bacteroidaceae (3.46\%), Prevotellaceae (3.41\%) and Peptostreptococcaceae (3.36\%). In addition, all samples shared two unclassified families belonging to class Clostridiales (0.13\%) and phyla Saccharibacteria (0.78\%).

Seven OTUs were classified to the genus Ruminococcaceae_UCG-005, comprising $13.34 \%$ of a total relative abundance, shared among all animals (Additional file 1: Table S2). OTUs belonging to Bacteroides (3.46\%), Christensenellaceae_R-7_group (2.62\%), Rikenellaceae_RC9_gut_group (2.78\%) and unclassified_Lachnospiraceae (2.66\%) each had more than five OTUs shared among all animals. The genera Acetitomaculum (0.14\%), Candidatus_Saccharimonas (0.78\%), Eubacterium_brachy_group (0.22\%), Mogibacterium (0.30\%), Prevotellaceae_UCG-001 (0.53\%), Prevotellaceae_UCG -003 (1.74\%), Ruminococcaceae_UCG-010 (0.29\%), Ruminococcaceae_UCG-013 (0.86\%) and unclassified_Prevotellaceae $(0.95 \%)$ each had two shared OTUs. Finally, there have 17 shared OTUs unclassified at the genus level (6.52\%), 8 of which were in Lachnospiraceae, 2 in Prevotellaceae, 3 in Ruminococcaceae, 1 in Peptococcaceae, 1 in Porphyromonadaceae, 1 in Bacteroidales_RF16_group, 1 in Bacteroidales_S24-7_group and 1 in Clostridiales.

\section{Lactation stages induced a variation in fecal bacteria community}

At the phylum level, the relative abundance of Firmicutes, Proteobacteria, Saccharibacteria and Cyanobacteria was significantly higher $(P<0.05)$ in the Fresh1d than in the Fresh14d group (Table 2). In contrast, Bacteroidetes and Tenericutes had higher $(P<0.01)$ relative abundance in Fresh14d compared with Fresh1d. Phyla Actinobacteria tended to increase $(P=0.067)$ and Verrucomicrobia tended to decrease $(P=0.067)$ as lactation progressed.

At the genus level, the relative abundance of Ruminococcaceae_UCG-005, Ruminococcaceae_UCG -013, Prevotellaceae_UCG-003, Alistipes, Ruminococcaceae_UCG-013 and Ruminococcaceae_UCG -014 was lower $(P<0.05)$ in the Fresh1d group compared with that in the Fresh14d group (Table 3). In contrast, the abundance of Christensenellaceae_R-7_ group, Clostridium_sensu_stricto_1, Romboutsia, Paeniclostridium, Lachnospiraceae_NK3A20_group, Escherichia-Shigella, Family_XIII_AD3011_group, Candidatus_Saccharimonas, Treponema_2 and Turicibacter was higher $(P<0.01)$ in the Fresh1d group than in the Fresh14d group. 


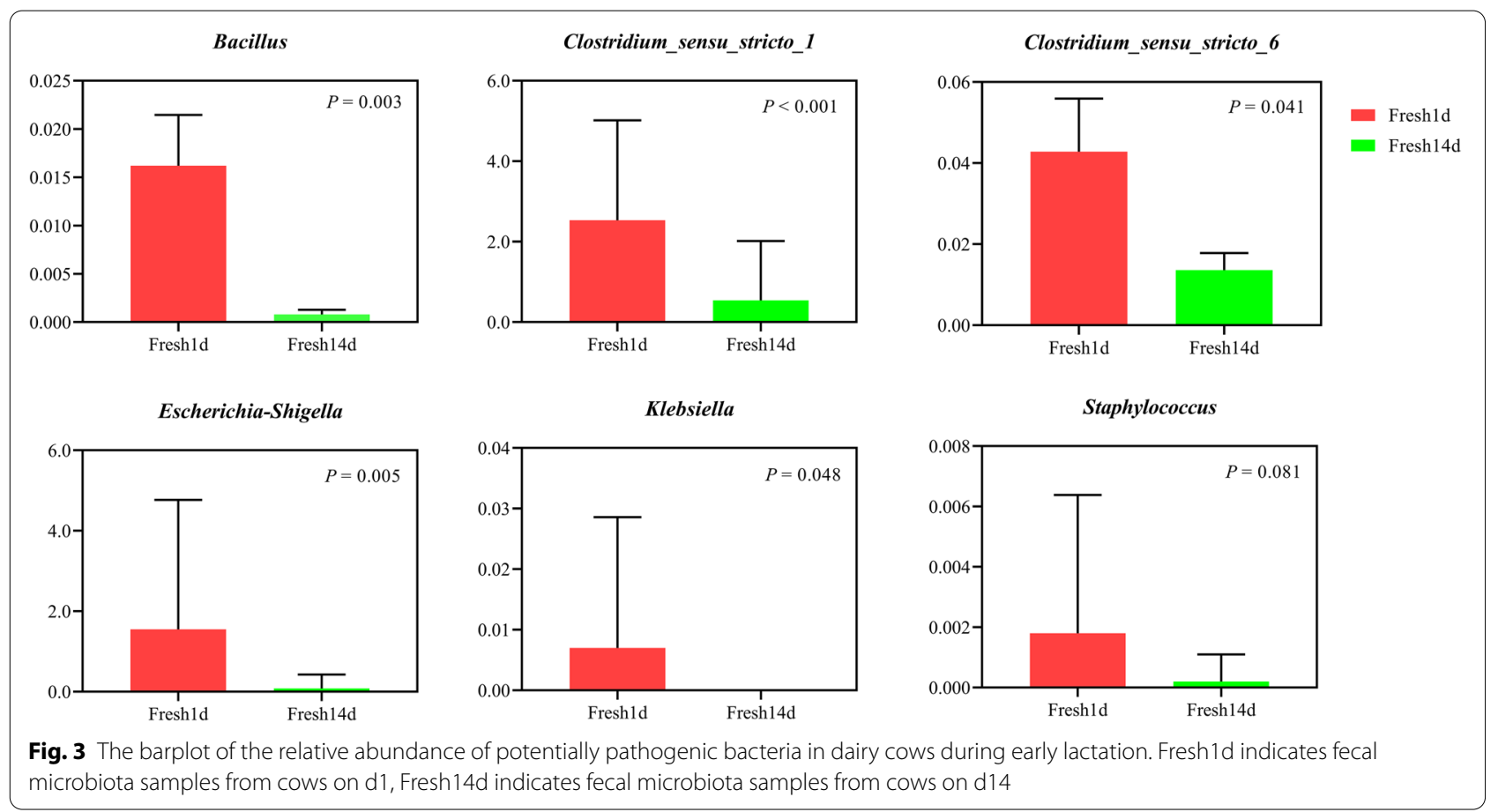

The abundance of some potentially pathogenic bacteria was significantly decreased $(P<0.05)$ as lactation progressed, these included Bacillus, Clostridium_sensu_ stricto_1, Clostridium_sensu_stricto_6 and EscherichiaShigella (Fig. 3). More specifically, the relative abundance of Klebsiella decreased from $0.007 \%$ on Fresh1d to $0 \%$ on Fresh14d ( $P=0.048$, Fig. 3). These findings suggest a higher risk of infection in dairy cows after calving and it was decreased as lactation progressed.

\section{Discussion}

The objective of this study was to elucidate the composition and the dynamic changes of the fecal microbiota in dairy cows during early lactation stage using $16 \mathrm{~S}$ rRNA gene sequencing technology. In this study, we found that the lactation stage of dairy cows significantly contributes to the variation in the fecal bacteria community of lactation cows. Lactation stage was significantly correlated to the relative abundances of the most major and minor contributing phyla. Clustering based on Bray-Curtis distances revealed that samples from Fresh1d clustered away from samples from Fresh14d. Additionally, Fresh1d samples had more OTUs, higher Chaol and Shannon index in common with Fresh14d samples. Collectively, our data demonstrate that the relative abundance of most major and minor contributing phyla differed by days post-freshening.

Similar to previous pyrosequencing-based studies (Hagey et al. 2019; Huang et al. 2020), the most abundant fecal bacteria at the phylum level that were shared across all samples were the Firmicutes and Bacteroidetes. We also found that the fecal bacteria community was dynamic over the lactation period, with large discrepancies were observed at the taxonomic level of the phylum and genus, indicating that the adult fecal microbial community with a fluctuation. Recently it was shown that lactation stage has a great impact on shaping the ruminal bacterial community in dairy cows (Bainbridge et al. 2016). Thus, the fecal bacteria difference of samples from Fresh1d and Fresh14d is understandable as they suffer the different lactation stages. To our best knowledge, no other studies have examined the shift in fecal bacterial populations with lactation, although some studies have described the dynamics of rumen bacteria during the transition period. For example, Pitta et al. (2014) described the temporal dynamics of rumen bacteria from five primiparous and five multiparous Holstein dairy cows during the transition period (21 days before parturition, 1-3, 28 and 56 days in milk). In their study, an increase in the abundance of bacteria in the phylum Bacteroidetes and a decrease in bacteria from the phylum Firmicutes were observed between pre- and post-partum periods in primiparous cows. In according to results from Pitta et al. (2014), a smilar results was observed in our study that an increase in the abundance of bacteria in the phylum Bacteroidetes and a decrease in bacteria from the phylum Firmicutes were observed from 1 to 14 days after calving. Another explanation is the dietary shift, as 
cows after calving experienced acute dietary transition and the microbiota adaption needs time. Before calving, cows were fed a low concentrate-to-forage diet and then switch to a high concentrate-to-forage diet. A large body of work points that the composition of the bovine foregut (Clemmons et al. 2019; Henderson et al. 2015) and hindgut microbiota (Kim et al. 2014; Zhang et al. 2018) was shaped by the diet; however, this study was not specifically designed to address the effects of specific feedstuffs on the bovine fecal microbiota. More studies are needed to research the effects of diet on fecal microbiota in lactation dairy cows.

In this study, we define the core taxa in feces as the 48 identified genera present in all samples of fresh cows. Of the two studies that investigated the fecal bacteria of dairy cows, one reported that genera Clostridium, Porphyromonas, Bacteroides, Ruminococcus, Alistipes, Lachnospira and Prevotella were shared in all samples of 20 lactating dairy cows (Dowd et al. 2008). The other study found a consistent presence of highly abundant generaPrevotella, CF231, YRC22, Parabacteroides, 5-7N15, Oscillospira, Ruminococcus, Clostridium, Mogibacterium, Coprococcus, Butyrivibrio, Dorea, Turicibacter and Treponema-in 150 lactating dairy cows (Hagey et al. 2019). While some families of all these genera are present in our core fecal bacteria, Paraprevotellaceae, Spirochaetaceae, Coriobacteriaceae, Turicibacteraceae and Mogibacteriaceae are not the part of our definition of core. Also, of the 13 genera shared in the 20 lactating dairy cows in their study, Bacteroides, Ruminococcus, Alistipes, Lachnospiraceae, Prevotella and Anaerotruncus were the core genera that we observed in 20 fresh cows. Together, these observations suggest that there may be a highly conserved core microbiota defined by the highly abundant genera for the fresh dairy cow feces. However, further studies are required to confirm and understand the function of the fecal microbiome in lactating dairy cows using metagenomic and transcriptomic methods.

Gram-positive rod-shaped Bacillus species can persist for many years in soil (Manyi-Loh et al. 2016) and cause clinical mastitis in dairy cows (Yu et al. 2006). The class Clostridia consists of gram-positive anaerobic spore-forming bacteria that are ubiquitous in the gastrointestinal tract (Girija et al. 2013). Some species of Clostridia have been linked with mastitis, blackleg, hemoglobinuria, malignant edema and infant botulism both in cattle and humans (Williams 2014). Streptococcus are gram-positive, facultatively anaerobic, lactic-acid producing commensal bacteria present in the gastrointestinal tract of humans and animals (Silva et al. 2011). Members of this genus can produce virulence factors and express antibiotic-resistance genes in fresh and dry cattle manure (Eaton and Gasson 2001; Franz et al. 2001).
In recent years, Klebsiella spp. mastitis has become a serious problem in herds because of using sand and dry feces bedding, this is attributed to fecal shedding of Klebsiella spp. by healthy adult dairy cows (Munoz et al. 2006). Klebsiella spp. is one of the main gram-negative pathogens (Olde Riekerink et al. 2008) that causes mastitis, a decrease in milk production, culling of dairy cows (Gröhn et al. 2004; Wilson et al. 2007) and antimicrobial resistance (Roberson et al. 2004). Similar to functional prediction, precise detection of pathogens was unfeasible with our data. Despite the fact that the relative abundance of genera commonly associated with pathogenic bacteria (genus Bacillus, Clostridium_sensu_stricto_1, Clostridium_sensu_stricto_6 and Escherichia-Shigella) decreased from $\mathrm{d} 1$ to $\mathrm{d} 14$ after freshening, we cannot say conclusively if these are pathogenic or commensal. Thus, PCR and culture-based methods remain the gold standard for routine detection of these pathogens.

In conclusion, we observed significant changes in alpha diversity and relative abundance of fecal microbial taxa at the phylum and genus level using $16 \mathrm{~S}$ rRNA gene amplicon sequencing analyses, in response to the dietary and lactation period perturbations encountered by dairy cows during early lactation period. Moreover, we found a decrease in genera associated with pathogenic bacteria as lactation progressed during early lactation onward. In accordance with a clear shift in the fecal bacteria after NMDS analyses, we observed significant changes in the relative abundance of the dominant phyla and genera in fecal bacteria as lactation progressed. Further research should focus on the impact of pathogenic and environmental factors on fecal microbial communities and the health of dairy cows.

\section{Supplementary information}

Supplementary information accompanies this paper at https://doi. org/10.1186/s13568-020-01106-3.

Additional file 1: Table S1. Ingredients and chemical composition of the experimental diets (as dry matter basis). Table S2. Shared bacterial OTUs and taxa among all samples.

\section{Acknowledgements}

We thank the staff at the Zhongdi Breeding Technology Company for daily animal care and milking. We also thank all members of Prof Shengli Li's laboratory for their assistance with sample collection.

\section{Authors' contributions}

H.S, J.S and W.F designed the experiment, S.H, W.F and H.J performed the experiment; H.S organized the database and performed the statistical analysis; H.S wrote and organized the manuscripts; H.S, G.M and L.S edited the manuscript; L.S provided funding support. All authors made a significant contribution to the development of the manuscript and approved the final version.

\section{Funding}

This work was supported by the National Natural Science Foundation of China grant number $31,772,628$, the China Agriculture System grant number 
CARS-36, National Key Research and Development Progarm of China grant number 2018YFD0501600.

\section{Availability of data and materials}

All the raw DNA sequences were deposited in the National Center for Biotechnology Information Sequence Read Archive database and are publicly accessible under the accession number PRJNA628713. The data set supporting the conclusions of this article is included within the article.

\section{Ethics approval and consent to participate}

This experiment and the study protocols were approved by the Institutional Animal Care and Use Committee of the China Agricultural University, China (CAU20161108-2)

\section{Consent for publication}

No applicable.

\section{Competing interests}

All authors declare no conflicts of interest.

\section{Author details}

${ }^{1}$ The State Key Laboratory of Animal Nutrition, Beijing Engineering Technology Research Center of Raw Milk Quality and Safety Control, College of Animal Science and Technology, China Agricultural University, Beijing 100193, China. ${ }^{2}$ College of Animal Science and Technology, Hebei Agricultural University, Baoding 071001, China. ${ }^{3}$ College of Animal Science and Technology, Yangzhou University, Yangzhou 225009, China.

Received: 15 June 2020 Accepted: 9 September 2020 Published online: 17 September 2020

\section{References}

Bainbridge ML, Cersosimo LM, Wright AD, Kraft J (2016) Rumen bacterial communities shift across a lactation in Holstein, Jersey and Holstein $\times$ Jersey dairy cows and correlate to rumen function, bacterial fatty acid composition and production parameters. FEMS Microbiol Ecol 92(5):fiw059. https ://doi.org/10.1093/femsec/fiw059

Behr C, Sperber S, Jiang X, Strauss V, Kamp H, Walk T, Herold M, Beekmann K, Rietjens I, van Ravenzwaay B (2018) Microbiome-related metabolite changes in gut tissue, cecum content and feces of rats treated with antibiotics. Toxicol Appl Pharmacol 355:198-210. https://doi.org/10.1016/j. taap.2018.06.028

Benjamini Y, Hochberg Y (1995) Controlling the false discovery rate: a practical and powerful approach to multiple testing. J Royal Statis Soc 57(1):289300. https://doi.org/10.1111/j.2517-6161.1995.tb02031.x

Bergmann GT (2017) Microbial community composition along the digestive tract in forage- and grain-fed bison. BMC Vet Res 13(1):253. https://doi. org/10.1186/s12917-017-1161-x

Caporaso JG, Kuczynski J, Stombaugh J, Bittinger K, Bushman FD, Costello EK, Fierer N, Peña AN, Goodrich JK, Gordon Jl, Huttely GA, Kelly ST, Knights D, Koenig JE, Ley RE, Lozupone CA, McDonald D, Muegge BD, Pirrybg M, Reeder J, Sevinsky JR, Trurnbaugh PJ, Walters WA, Widmann J, Yatsunenko T, Zaneveld J, Knight R (2010) QIIME allows analysis of highthroughput community sequencing data. Nat Methods 7:335-336. https://doi. org/10.1038/nmeth0510-335

Caporaso JG, Lauber CL, Walters WA, Berg-Lyons D, Huntley J, Fierer N, Owens SM, Betley J, Fraser L, Bauer M, Gormley N, Gilbert JA, Smith G, Knight R (2012) Ultra-high-throughput microbial community analysis on the Illumina HiSeq and MiSeq platforms. ISME J 6(8):1621-1624. https://doi. org/10.1038/ismej.2012.8

Clemmons BA, Voy BH, Myer PR (2019) Altering the gut microbiome of cattle: considerations of host-microbiome interactions for persistent microbiome manipulation. Microbial Ecol 77:523-536. https://doi.org/10.1007/ s00248-018-1234-9

Dill-McFarland KA, Weimer PJ, Breaker JD, Suen G (2019) Diet influences early microbiota development in dairy calves without long-term impacts on milk production. Appl Environ Microbiol 85(2):e02141. https://doi. org/10.1128/AEM.02141-18
Dowd SE, Callaway TR, Wolcott RD, Sun Y, McKeehan T, Hagevoort RG, Edrington TS (2008) Evaluation of the bacterial diversity in the feces of cattle using 16S rDNA bacterial tag-encoded FLX amplicon pyrosequencing (bTEFAP). BMC Microbiol 8:125. https://doi.org/10.1186/1471-2180-8-125

Eaton TJ, Gasson MJ (2001) Molecular screening of Enterococcus virulence determinants and potential for genetic exchange between food and medical isolates. Appl Environ Microbiol 67(4):1628-1635. https://doi. org/10.1128/AEM.67.4.1628-1635.2001

Edgar RC (2013) UPARSE: highly accurate OTU sequences from microbial amplicon reads. Nat Methods 10(10):996-998. https://doi.org/10.1038/ nmeth.2604

Flint HJ, Bayer EA, Rincon MT, Lamed R, White BA (2008) Polysaccharide utilization by gut bacteria: potential for new insights from genomic analysis. Nat Rev Microbiol 6(2):121-131. https://doi.org/10.1038/nrmicro1817

Franz CMAP, Muscholl-Silberhorn AB, Yousif NMK, Vancanneyt M, Swings J, Holzapfel WH (2001) Incidence of virulence factors and antibiotic resistance among Enterococci isolated from food. Appl Environ Microbiol 67(9):4385-4389. https://doi.org/10.1128/AEM.67.9.4385-4389.2001

Girija D, Deepa K, Xavier F, Antony I, Shidhi P (2013) Analysis of cow dung microbiota-a metagenomic approach. Indian J Biotechnol 12:372-378

Gomez DE, Arroyo LG, Costa MC, Viel L, Weese JS (2017) Characterization of the fecal bacterial microbiota of healthy and diarrheic dairy calves. J Vet Int Med 31(3):928-939. https://doi.org/10.1111/jvim.14695

Gröhn YT, Wilson DJ, González RN, Hertl JA, Schulte JH, Bennett G, Schukken YH (2004) Effect of pathogen-specific clinical mastitis on milk yield in dairy cows. J Dairy Sci 87:3358-3374. https://doi.org/10.3168/jds.S0022 -0302(04)73472-4

Hagey JV, Bhatnagar S, Heguy JM, Karle BM, Price PL, Meyer D, Maga EA (2019) Fecal microbial communities in a large representative cohort of california dairy cows. Front Microbiol 10:1093. https://doi.org/10.3389/fmicb 2019.01093

Henderson G, Cox F, Ganesh S, Jonker A, Young W, Collaborators GRC, Janssen $\mathrm{PH}$ (2015) Rumen microbial community composition varies with diet and host, but a core microbiome is found across a wide geographical range. Sci Rep 5:14567. https://doi.org/10.1038/srep14567

Huang S, Ji SK, Yan H, Hao YY, Zhang J, Wang YJ, Cao ZJ, Li SL (2020) The day-today stability of the ruminal and fecal microbiota in lactating dairy cows. Microbiologyopen 9(5):e990. https://doi.org/10.1002/mbo3.990

Indugu N, Vecchiarelli B, Baker LD, Ferguson JD, Vanamala JKP, Pitta DW (2017) Comparison of rumen bacterial communities in dairy herds of different production. BMC Microbiol 17(1):190. https://doi.org/10.1186/s1286 6-017-1098-z

Kim M, Kim J, Kuehn LA, Bono JL, Berry ED, Kalchayanand N, Freetly HC, Benson AK, WElls JE (2014) Investigation of bacterial diversity in the feces of cattle fed different diets. J Anim Sci 92(2):683-694. https://doi.org/10.2527/ jas2013-6841

Lima FS, Oikonomou G, Lima SF, Bicalho ML, Ganda EK, Filho JC, Lorenzo G, Trojacanec P, Bicalhoa RC (2015) Prepartum and postpartum rumen fluid microbiomes: characterization and correlation with production traits in dairy cows. Appl Environ Microbiol 81(4):1327-1337. https://doi. org/10.1128/AEM.03138-14

Manyi-Loh CE, Mamphweli SN, Meyer EL, Makaka G, Simon M, Okoh Al (2016) An overview of the control of bacterial pathogens in cattle manure. Int J Environ Res Public Health 13(9):843. https://doi.org/10.3390/ijerph1309 0843

Munoz MA, Ahlström C, Rauch BJ, Zadoks RN (2006) Fecal shedding of Klebsiella pneumoniae by dairy cows. J Dairy Sci 89:3425-3430. https://doi. org/10.3168/jds.S0022-0302(06)72379-7

Myer PR, Wells JE, Smith TP, Kuehn LA, Freetly HC (2015) Cecum microbial communities from steers differing in feed efficiency. J Anim Sci 93(11):5327. https://doi.org/10.2527/jas2015-9415

Oksanen J, Blanchet FG, Kindt R, Legendre P, Minchin PR, O'Hara RB, Simpson GL, Solymos P, Stevens MHH (2015) Vegan: community ecology package 2.2-1

Olde Riekerink RG, Barkema HW, Kelton DF, Scholl DT (2008) Incidence rate of clinical mastitis on Canadian dairy farms. J Dairy Sci 91(4):1366-1377. https://doi.org/10.3168/jds.2007-0757

Oultram J, Phipps E, Teixeira AG, Foditsch C, Bicalho ML, Machado VS, Bicalho RC, Oikonomou G (2015) Effects of antibiotics (oxytetracycline, florfenicol or tulathromycin) on neonatal calves' faecal microbial diversity. Vet Rec 177(23):598. https://doi.org/10.1136/vr.103320 
Pitta DW, Kumar S, Vecchiarelli B, Shirley DJ, Bittinger K, Baker LD (2014) Temporal dynamics in the ruminal microbiome of dairy cows during the transition period. J Anim Sci 92(9):4014-4022. https://doi.org/10.2527/ jas2014-7621

Pruesse E, Quast C, Knittel K, Fuchs BM, Ludwig W, Peplies J, Glockner FO (2007) SILVA: a comprehensive online resource for quality checked and aligned ribosomal RNA sequence data compatible with ARB. Nucleic Acids Res 35(21):7188-7196. https://doi.org/10.1093/nar/gkm864

Roberson JR, Warninck LD, Moore G (2004) Mild to moderate clinical mastitis efficacy of intramammary amoxicillin. J Dairy Sci 87:583-592. https://doi. org/10.3168/jds.S0022-0302(04)73200-2

Shabat SK, Sasson G, Doron-Faigenboim A, Durman T, Yaacoby S, Berg Miller ME, White BA, Shterzer N, Mizrahi I (2016) Specific microbiome-dependent mechanisms underlie the energy harvest efficiency of ruminants. ISME J 10(12):2958-2972. https://doi.org/10.1038/ismej.2016.62

Silva N, Igrejas G, Gonçalves A, Poeta P (2011) Commensal gut bacteria: distribution of Enterococcus species and prevalence of Escherichia coli phylogenetic groups in animals and humans in Portugal. Annals Microbiol 62(2):449-459. https://doi.org/10.1007/s13213-011-0308-4

Song ES, Jung SI, Park HJ, Seo KW, Son JH, Hong S, Shim M, Kim HB, Song KH (2016) Comparison of fecal microbiota between german holstein dairy cows with and without left-sided displacement of the Abomasum. J Clin Microbiol 54(4):1140-1143. https://doi.org/10.1128/JCM.02442-15

Song Y, Malmuthuge N, Steele MA, Guan LL (2018) Shift of hindgut microbiota and microbial short chain fatty acids profiles in dairy calves from birth to pre-weaning. FEMS Microbiol Ecol 94(3):1-15. https://doi.org/10.1093/ femsec/fix179

Tang MT, Han H, Yu Z, Tsuruta T, Nishino N (2017) Variability, stability, and resilience of fecal microbiota in dairy cows fed whole crop corn silage. Appl Microbiol Biotech 101(16):6355-6364. https://doi.org/10.1007/s0025 3-017-8348-8

Tong J, Zhang H, Yang D, Zhang Y, Xiong B, Jiang L (2018) Illumina sequencing analysis of the ruminal microbiota in high-yield and low-yield lactating dairy cows. PLoS ONE 13(11):e0198225. https://doi.org/10.1371/journ al.pone.0198225

Wang Q, Garrity GM, Tiedje JM, Cole JR (2007) Naive Bayesian classifier for rapid assignment of rRNA sequences into the new bacterial taxonomy. Appl Environ Microbiol 73(16):5261-5267. https://doi.org/10.1128/AEM.00062 $-07$

Wang B, Ma MP, Diao QY, Tu Y (2019) Saponin-induced shifts in the rumen microbiome and metabolome of young cattle. Front Microbiol 10:356. https://doi.org/10.3389/fmicb.2019.00356
Weimer PJ, Cox MS, Vieira de Paula T, Lin M, Hall MB, Suen G (2017) Transient changes in milk production efficiency and bacterial community composition resulting from near-total exchange of ruminal contents between high- and low-efficiency Holstein cows. J Dairy Sci 100(9):7165-7182. https://doi.org/10.3168/jds.2017-12746

Williams S (2014) Health Risks from Pathogens in Livestock Manure. Waste contaminants: life cycle and entry into food chain. sustainable organic waste management for environmental protection and food safety. http:// ramiran.uvlf.sk/doc04/SESSION\%203.pdf. Accessed 28 May 2014

Wilson DJ, Grohn YT, Bennett GJ, Gonzalez RN, Schukken YH, Spatz J (2007) Comparison of J5 vaccinates and controls for incidence, etiologic agent, clinical severity, and survival in the herd following naturally occurring cases of clinical mastitis. J Dairy Sci 90(9):4282-4288. https://doi. org/10.3168/jds.2007-0160

Xue M, Sun H, Wu X, Guan LL, Liu J (2018) Assessment of rumen microbiota from a large dairy cattle cohort reveals the pan and core bacteriomes contributing to varied phenotypes. Appl Environ Microbiol 84(19):e00970. https://doi.org/10.1128/AEM.00970-18

Yousif MH, Li JH, Li ZQ, Maswayi Alugongo G, Ji SK, Li YX, Wang YJ, Li SL, Cao ZJ (2018) Low concentration of antibiotics modulates gut microbiota at different levels in pre-weaning dairy calves. Microorganisms 6(4):118. https ://doi.org/10.3390/microorganisms6040118

Yu ZT, Yu M, Morrison M (2006) Improved serial analysis of V1 ribosomal sequence tags (SARST-V1) provides a rapid, comprehensive, sequencebased characterization of bacterial diversity and community composition. Environ Microbiol 8:603-611. https://doi.org/10.3168/jds.S0022 $-0302(04) 73472-4$

Zhang JC, Xu C, Huo D, Hu Q, Peng Q (2017) Comparative study of the gut microbiome potentially related to milk protein in Murrah buffaloes (Bubalus bubalis) and Chinese Holstein cattle. Sci Rep 7:42189. https://doi. org/10.1038/srep42189

Zhang J, Shi HT, Wang YJ, Cao ZJ, Yang HJ, Li SL (2018) Effect of limit-fed diets with different forage to concentrate ratios on fecal bacterial and archaeal community composition in holstein heifers. Front Microbiol 9:976. https ://doi.org/10.3389/fmicb.2018.00976

\section{Publisher's Note}

Springer Nature remains neutral with regard to jurisdictional claims in published maps and institutional affiliations.

\section{Submit your manuscript to a SpringerOpen ${ }^{\circ}$ journal and benefit from:}

- Convenient online submission

- Rigorous peer review

- Open access: articles freely available online

- High visibility within the field

- Retaining the copyright to your article

Submit your next manuscript at springeropen.com 